\title{
Algorithms for Calculating the Limits of Convergent Infinite Series
}

\author{
Héctor Luna-García*, Luz María García-Cruz and Ana Elizabeth García \\ (Communicated by İ. Onur KIYMAZ)
}

\begin{abstract}
We use algorithms to calculate the exact limits of a wide range of convergent infinite series by means of special functions, these are polygamma functions.

However, in the case of alternating series, these algorithms do not allow the use of such functions, but allow them to find the limits of this series. Finally, these methods are used as a powerful and simple tool for calculating the limits of many infinite series as shown in the examples included.

Keywords: Convergent infinite series; Polygamma functions; Laplace transform.

AMS Subject Classification (2010): Primary: 40A30 ; Secondary: 33B15; 44A10.

${ }^{*}$ Corresponding author
\end{abstract}

\section{Introduction}

Infinite series are in physics from simple geometric $\sum_{n=1}^{\infty} a r^{n}$ in black body radiation, as $\sum_{n=1}^{\infty} \frac{1}{(2 n-1)(2 n+1)(2 n+3)}$ which lies in creating neutrons (by a nuclear reaction) into hollow sphere. Therefore it is necessary to have knowledge of infinite series in a course in calculus with applications. To consider the infinite series in the literature is natural to introduce some convergence criteria due to Cauchy, Kummer, D'Alambert, and Gauss authors [1], which ensure us the convergence of infinite series. In addition, in the alternating series there are some criteria or test that help quickly conclude its convergence, ration test and root criteria [2].However, the application of these criteria do not allow us to know their limits. In this paper, we present some algorithms to compute the exact limit of a broad class of infinite series which converge. This work is divided in five parts: This introduction, in section 2 the method is illustrated with some special examples; in section 3 the method is generalized and Laplace transform is used for certain types of series; in section 4 the authors present the infinite series alternating. Finally, section 5 present conclusions and future projects.

\section{Cases of Infinite Series}

2.1 The Infinite series $S(a, b)=\sum_{n=1}^{\infty} \frac{1}{(n+a)(n+b)}$.

In this series $S(a, b)$, we have $a$ and $b \in \mathbb{Z}^{+}$or positive fractional numbers and it is decomposed into partial fractions as

Received : 29-September-2015, Accepted : 30-December-2015 


$$
S(a, b)=\sum_{n=1}^{\infty}\left(\frac{1}{(n+a)}-\frac{1}{n+b}\right) \frac{1}{(b-a)}
$$

and using the Feynman's identity

$$
\frac{1}{A}=\int_{0}^{\infty} e^{-A x} d x
$$

where $A>0$, Eq. (2.1) becomes

$$
S(a, b)=\frac{1}{(a-b)} \sum_{n=1}^{\infty} \int_{0}^{\infty} e^{-n x}\left(e^{-b x}-e^{-a x}\right) d x .
$$

Introducing the sum into the integral in Eq.(2.3), one has

$$
S(a, b)=\frac{1}{(a-b)} \int_{0}^{\infty}\left(e^{-b x}-e^{-a x}\right) e^{-x} \lim _{N \rightarrow \infty} \sum_{n=1}^{\infty} \frac{\left(e^{-x}-e^{-N x}\right)}{\left(1-e^{-x}\right)} d x .
$$

Considering now $N \rightarrow \infty$ and the properties of geometric series, the result is

$$
S(a, b)=\frac{1}{(a-b)} \sum_{n=1}^{\infty} \int_{0}^{\infty}\left(e^{-b x}-e^{-a x}\right) \frac{e^{-x}}{1-e^{-x}} d x .
$$
as:

Changing $z=e^{-x}$ variables and using the polygamma functions properties [3], the Eq. (2.5) finally is expressed

$$
S(a, b)=\frac{1}{(a-b)} \int_{0}^{1} \frac{z^{b}-z^{a}}{1-z} d z=\frac{\psi(b+1)-\psi(a+1)}{b-a}=\frac{\psi(b)-\psi(a)}{b-a}-\frac{1}{b a}
$$

where $\psi(z) \doteq \psi^{(0)}(z)$ is the called digamma function.

Eq.(2.6) is the most significant expression in this section. As examples of the present application we have

$$
\begin{gathered}
\sum_{n=1}^{\infty} \frac{1}{(n+1)(n+2)}=\frac{1}{2}, \\
\sum_{n=1}^{\infty} \frac{1}{n\left(n+\frac{1}{2}\right)}=2(2-\ln 2) .
\end{gathered}
$$

2.2 The infinite series with $a=b$

In this case is applied the Feynman's identity

$$
\frac{1}{A^{2}}=\int_{0}^{\infty} x e^{-A x} d x
$$

Following the same procedure in section 2.1, leads to the following result.

$$
\sum_{n=1}^{\infty} \frac{1}{(n+a)^{2}}=\int_{0}^{\infty} \frac{x e^{-(a+1)}}{1-e^{-x}} d x=\int_{0}^{1} \frac{z^{a} \ln z}{1-z} d z=\psi^{(1)}(a+1),
$$

where $\psi^{(1)}(x)$ is the poligamma function of order one. The Eq. (2.10) is now wxpressed in terms of $\psi^{(n)}(x)$ polygamma function [3].

Applying Eq. (2.10) already reported results are obtained 


$$
\begin{gathered}
\sum_{n=1}^{\infty} \frac{1}{n^{2}}=\psi^{(1)}(1)=\frac{\pi^{2}}{6}, \\
\sum_{n=1}^{\infty} \frac{1}{(n+1)^{2}}=\psi^{(1)}(2)=\frac{\pi^{2}}{6}-1 .
\end{gathered}
$$

\section{Generalization of infinite series}

To generalize the infinite series sofar treated, let us consider the series denoted as $S\left(a^{m}\right)=\sum_{n=1}^{\infty} \frac{1}{(n+a)^{m}}$, and apply the most general Feynman's identity

$$
\frac{1}{A^{m}}=\frac{1}{(m-1) !} \int_{0}^{\infty} x^{m-1} e^{-A x} d x
$$

And folowing the same procedure of previous, the series can be written as

$$
\sum_{n=1}^{\infty} \frac{1}{(n+a)^{m}}=\frac{(-1)^{m}}{(m-1) !} \int_{0}^{1} \frac{z^{a}(\ln z)^{m-1}}{1-z} d z=\frac{(-1)^{m}}{(m-1) !} \psi^{(m-1)}(a+1) .
$$

\subsection{Infinite series with conjugated binomials}

Let us consider the following infinite series

$$
S(a)=\sum_{n=1}^{\infty} \frac{1}{(n-a)(n+a)}
$$

where $a<1$ is a fractional number.

The $S(a)$ term is descomposed into two fractions

$$
S(a)=\frac{1}{2 a} \sum_{n=1}^{\infty}\left(\frac{1}{n-a}-\frac{1}{n+a}\right)
$$

Following similar procedures to those already made, it finally reaches the value of the infinite series in terms of digamma function

$$
S(a)=\frac{1}{2 a} \int_{0}^{1} \frac{z^{a}-z^{-a}}{1-z} d z=\frac{1}{2 a}[\psi(1-a)-\psi(1+a)]
$$

Applying Eq. (3.5) and the properties of the polygamma functions, we have

$$
\begin{gathered}
\sum_{n=1}^{\infty} \frac{1}{(8 n-1)(8 n+1)}=\frac{1}{64} \sum_{n=1}^{\infty} \frac{1}{\left(n-\frac{1}{8}\right)\left(n+\frac{1}{8}\right)}=\frac{1}{64}[32-4 \pi(\sqrt{2}-1)] \\
=\frac{1}{2}-\frac{\pi}{16}(\sqrt{2}-1)
\end{gathered}
$$

Also,

$$
\sum_{n=1}^{\infty} \frac{1}{(4 n-1)(4 n+1)}=\frac{1}{16} \sum_{n=1}^{\infty} \frac{1}{\left(n-\frac{1}{4}\right)\left(n+\frac{1}{4}\right)}=\frac{1}{16}(32-2 \pi)=\frac{1}{2}-\frac{\pi}{8} .
$$

As another example, we find

$$
\sum_{n=1}^{\infty} \frac{1}{(2 n-1)(2 n+1)}=\frac{1}{4} \sum_{n=1}^{\infty} \frac{1}{\left(n-\frac{1}{2}\right)\left(n+\frac{1}{2}\right)}=\frac{1}{4}(2)=\frac{1}{2} .
$$

These series are found in Ref [4] . 


\subsection{Infinite series and Laplace transform}

As shown, Feynman's identities given by Eqs. (2.2), (2.9), and (3.1) are the Laplace's transforms of $1, x, x^{m-1}$, respectively. In general, if $g(s)$ is the Laplace's transform of $f(x)$, one has

$$
g(s)=\int_{0}^{\infty} e^{-s x} f(x) d z
$$

where the sum $\sum_{n} g(n)$ can be written as

$$
\sum_{n} g(n)=\int_{0}^{\infty} f(x)\left(\sum_{n} e^{-n x}\right) d x
$$

where the sum and integral operations are exchanged. Now, let us present two examples of the applications of the Laplace's transform.

For the first example, the following expression is considered

$$
\frac{s}{(s+a)(s+b)}=L\left(\frac{a e^{-a x}-b e^{-b x}}{a-b}\right) .
$$

Then, the infinite series associated with this expression is given by

$$
\sum_{n=1}^{\infty} \frac{n}{(n+a)(n+b)}=\sum_{n} \int_{0}^{\infty} e^{-n x}\left(\frac{a e^{-a x}-b e^{-b x}}{a-b}\right) d x
$$

which becomes

$$
\sum_{n=1}^{\infty} \frac{n}{(n+a)(n+b)}=\frac{1}{a-b} \int_{0}^{1} \frac{a z^{a}-b z^{b}}{1-z} d z,
$$
is

where $z=e^{-x}$, the variable change is used. Applying the digamma function properties, the infinite series limit

$$
\sum_{n=1}^{\infty} \frac{n}{(n+a)(n+b)}=\frac{b \psi(b+1)-a \psi(a+1)}{a-b}=\frac{b \psi(b)-a \psi(a)}{a-b}-\frac{1}{a b} .
$$

For the second example, the following series is used $\sum_{n=1}^{\infty} \frac{\Gamma(k)}{(n+a)^{k}}$, where $\Gamma(x)$ it is the well-known gamma function.

Since, the term in the summation is the Laplace's transform of $x^{k-1} e^{-a x}$, and applying our method directly, the results is

$$
\begin{gathered}
\sum_{n=1}^{\infty} \frac{\Gamma(k)}{(n+a)^{k}}=\sum_{n=1}^{\infty} \int_{0}^{\infty} e^{-n x} x^{k-1} e^{-a x} d x=(-1)^{k-1} \int_{0}^{1} \frac{z^{a}(\ln z)^{k-1}}{1-z} d z \\
=(-1)^{k} \psi^{(k-1)}(a+1),
\end{gathered}
$$

where again $z=e^{-x}$, the variable change is used.

To introduce section of alternating series, consider the following Leibnitz expression $\sum_{n=0}^{\infty} \frac{(-1)^{n}}{2 n+1}$. Using the method of section 2,

$$
\sum_{n=1}^{\infty} \frac{(-1)^{n}}{2 n+1}=\int_{0}^{\infty} e^{-x} \sum_{n=1}^{\infty}\left[(-1)^{n} e^{-2 n x}\right] d x
$$

and since the series $\sum_{n=1}^{\infty}(-1)^{n} e^{-2 n x}$ can be also evaluated, finally one has 


$$
\sum_{n=1}^{\infty} \frac{(-1)^{n}}{2 n+1}=\frac{1}{2} \int_{0}^{\infty} \frac{e^{-\frac{3 x}{2}}}{1+e^{-2 x}} d x=\int_{1}^{0} \frac{w^{2}}{1+w^{2}} d w=\frac{\pi}{4}-1, \text { then } \sum_{n=0}^{\infty} \frac{(-1)^{n}}{2 n+1}=\frac{\pi}{4} .
$$

However, there are other cases as important as this series and we present these cases.

\section{Alternating Series}

4.1 Series of the form $S(a)=\sum_{n=1}^{\infty} \frac{(-1)^{n-1}}{a^{n}}$ where $b>1$ and is an integer.

Using the first Feynman's identity given for Eq. (2.2), the series $S(a)$ becomes

$$
S(a)=\sum_{n=1}^{\infty} \int_{0}^{\infty}(-1) e^{-a^{n} x} d x .
$$

Introducing the sum into the integral in Eq. (4.1), and changing $y=e^{-a^{n} x}$ variables, one has

$$
S(a)=\sum_{n=1}^{\infty} \frac{(-1)^{n-1}}{a^{n}}=\int_{1}^{0} \sum_{n=1}^{\infty}\left(-\frac{1}{a}\right)^{n} d y=\int_{1}^{0} \lim _{N \rightarrow \infty} \sum_{n=1}^{\infty} \frac{\left(-\frac{1}{a}\right)-\left(-\frac{1}{a}\right)^{N}}{1-\left(-\frac{1}{a}\right)} d y .
$$

Considering now $N \rightarrow \infty$, the result is

$$
S(a)=\sum_{n=1}^{\infty} \frac{(-1)^{n-1}}{a^{n}}=\int_{0}^{1} \frac{1}{1+a} d y=\frac{1}{1+a} .
$$

As an application, we present the following examples:

$$
\sum_{n=0}^{\infty} \frac{(-1)^{n}}{2^{n}}=\frac{2}{3}, \sum_{n=0}^{\infty} \frac{(-1)^{n-1}}{5^{n}}=-\frac{5}{6}
$$

4.2 Series of the form $S(2 n, a)=\sum_{n=1}^{\infty} \frac{(-1)^{n}}{a^{2 n}}$.

Following the same algorithm as in section 4.1, we found

$$
S(2 n, a)=\sum_{n=1}^{\infty} \frac{(-1)^{n}}{a^{2 n}}=-\frac{1}{\left(1+a^{2}\right)}
$$

with the following examples

$$
\sum_{n=1}^{\infty} \frac{(-1)^{n}}{3^{2 n}}=-\frac{1}{10}, \sum_{n=0}^{\infty} \frac{(-1)^{n}}{2^{2 n}}=\frac{4}{5}
$$

4.3 Series of the form $S(n, a)=\sum_{n=1}^{\infty} \frac{(-1)^{n}}{n+a}$.

For this series, one has

$$
S(n, a)=\sum_{n=1}^{\infty}(-1)^{n} \int_{0}^{\infty} e^{-(n+a) x} d x
$$

where we have used the Feymnan's identity.

To Eq. (4.6), using the same procedure, one has

$$
S(n, a)=\int_{0}^{\infty} e^{-a x} \sum_{n=1}^{\infty}\left(-e^{-x}\right)^{n} d x=\int_{0}^{\infty} e^{-a x} \lim _{N \rightarrow \infty} \frac{\left(-e^{-x}\right)-\left(-e^{-x}\right)^{N}}{1-\left(-e^{-x}\right)} d x .
$$


Then, in the limit as $N \rightarrow \infty$, the result is

$$
S(n, a)=\sum_{n=1}^{\infty} \frac{(-1)^{n}}{n+a}=\int_{0}^{\infty} \frac{e^{-a x}\left(-e^{-x}\right)}{1+e^{-x}} d x=\int_{1}^{0} \frac{u^{a}}{1+u} d u,
$$

where $z=e^{-x}$, the variable change was used.

At this point, it is important to mention that integral given by Eq. (4.8), can not be written in terms of poligamma functions, due to the plus sign in the binomial denominator. In contrast to those founded in section 2 . However, this equation is the most general expression result of this section. As one application, we present the following examples:

$$
\sum_{n=1}^{\infty} \frac{(-1)^{n+1}}{n}=\ln 2, \sum_{n=1}^{\infty} \frac{(-1)^{n+1}}{3 n-2}=\frac{1}{3}\left(\ln 2+\frac{\pi}{\sqrt{3}}\right)
$$

and,

$$
\sum_{n=1}^{\infty} \frac{(-1)^{n+1}}{4 n-3}=\frac{1}{4 \sqrt{2}}(\pi+2 \ln (\sqrt{2}+1)) .
$$

This series are found in Ref. [4].

4.4 Series of the form $S(2, a)=\sum_{n=1}^{\infty} \frac{(-1)^{n+1}}{(n+a)^{2}}$.

Using Feynman's identity and folowing the same procedure as was used in section 2, leads to the result

$$
S(2, a)=\sum_{n=1}^{\infty} \frac{(-1)^{n+1}}{(n+a)^{2}}=\int_{0}^{\infty} \frac{x e^{-(a+1)}}{1+e^{-x}} d x=\int_{1}^{0} \frac{(\ln z) z^{a}}{1+z} d z,
$$

wher again $z=e^{-x}$.

Applying Eq. (4.11) the already reported result is obtained

$$
\sum_{n=1}^{\infty} \frac{(-1)^{n+1}}{n^{2}}=\frac{\pi^{2}}{12}
$$

4.5 Type series $S(2 k, n)=\sum_{n=1}^{\infty} \frac{(-1)^{n-1}}{n^{2 k}}$, with $k=1,2, \ldots$

Using Feynman's identity for $\frac{1}{A^{2}}$, and applied to this case the same procedure as was used in section 2, leads to the result

$$
S(2 k, n)=\sum_{n=1}^{\infty} \frac{(-1)^{n-1}}{n^{2 k}}=\frac{1}{(2 k-1) !} \int_{0}^{\infty} \frac{x^{2 k-1} e^{-x}}{1+e^{-x}} d x=\frac{1}{(2 k-1) !} \int_{0}^{1} \frac{(-\ln z)^{2 k-1}}{1+z} d z .
$$

Applying Eq. (4.13), we have

$$
\sum_{n=1}^{\infty} \frac{(-1)^{n-1}}{n^{2}}=\frac{\pi^{2}}{12}
$$

whose value was found in section 2. As another examples, we find

$$
\sum_{n=1}^{\infty} \frac{(-1)^{n-1}}{n^{4}}=\frac{1}{6} \int_{1}^{0} \frac{(\ln z)^{3}}{1+z}=\frac{1}{6}\left(\frac{7 \pi^{4}}{120}\right)=\frac{7}{720} \pi^{4}
$$

and, 


$$
\sum_{n=1}^{\infty} \frac{(-1)^{n-1}}{n^{6}}=\frac{1}{120} \int_{1}^{0} \frac{(\ln z)^{5}}{1+z}=\frac{1}{120}\left(\frac{31 \pi^{6}}{252}\right)=\frac{31}{30240} \pi^{6} .
$$

These series are found in Ref. [4].

4.6 Type series $S(2 k+1, n)=\sum_{n=1}^{\infty} \frac{(-1)^{n-1}}{(2 n-1)^{2 k+1}}$, with $k=1,2, \ldots$

Following the same procedure, leads to the following result

$$
S(2 k+1, n)=\frac{1}{2^{k}(2 k) !} \int_{0}^{\infty} \frac{x^{2 k} e^{-\frac{x}{2}}}{1+e^{-x}} d x=\frac{1}{2^{k}(2 k) !} \int_{0}^{\infty} \frac{(\ln z)^{2 k} z^{-\frac{1}{2}}}{1+z} d z
$$

Changing $z=w^{2}$ variables, Eq. (4.16) finally is expressed as

$$
S(2 k+1, n)=\sum_{n=1}^{\infty} \frac{(-1)^{n-1}}{(2 n-1)^{2 k+1}}=\frac{1}{2^{k}(2 k) !} \int_{0}^{1} \frac{\left(\ln w^{2}\right)^{2 k}}{1+w^{2}} d w .
$$

Finally, one has the following examples:

$$
\begin{gathered}
\sum_{n=1}^{\infty} \frac{(-1)^{n-1}}{(2 n-1)^{3}}=\frac{1}{8} \int_{0}^{1} \frac{\left(\ln w^{2}\right)^{2}}{1+w^{2}} d w=\frac{1}{8}\left(\frac{\pi^{3}}{4}\right)=\frac{\pi^{3}}{32} \\
\sum_{n=1}^{\infty} \frac{(-1)^{n-1}}{(2 n-1)^{5}}=\frac{1}{384} \int_{0}^{1} \frac{\left(\ln w^{2}\right)^{4}}{1+w^{2}} d w=\frac{1}{384}\left(\frac{5 \pi^{5}}{4}\right)=\frac{\pi^{5}}{1536},
\end{gathered}
$$

and,

$$
\sum_{n=1}^{\infty} \frac{(-1)^{n-1}}{(2 n-1)^{7}}=\frac{1}{46080} \int_{0}^{1} \frac{\left(\ln w^{2}\right)^{6}}{1+w^{2}} d w=\frac{1}{46080}\left(\frac{61 \pi^{7}}{4}\right)=\frac{\pi^{7}}{184320} .
$$

These series are found in Ref. [4]. It is noteworthy that the above integrals can be solved by contour integration.

\section{Conclusions}

The limits of a broad class of convergent infinite series were obtained using some novel algorithms, no matter whether are alternating or not. Thes algorithms are very flexible and can be used to evaluate many series that are important in different physics disciplines, as well as in other relevant sciences applications. Furthermore, not all algorithms used for no alternating series can be used for the alternating (polygamma functions). However, all the algoritms shown in this paper can be used for each type of series.

\section{References}

[1] Arfken, G., Weber, H., and Harris, F., Mathematical Methods for Physicists: A Comprehensive Guide, Elsevier Academic Press, USA, 2012.

[2] Spivak. M., Calculus, Editorial Reverté, España, 1992.

[3] Abramowitz, M., Stegun, I., Handbook of Mathematical Functions: with Formulas, Graphs, and Mathematical Tables, Dover Publications, USA, 1964.

[4] Gradshteyn, I., Ryzhik, I., Jeffrey, A., Zwillinger, D., Table of Integrals, Series and Products, Academic Press, USA, 2000. 


\section{Affiliations}

HÉCTOR LUNA GARCÍA

AdDress: Autonomous Metropolitan University, Dept. of Basic Sciences, 0200, Mexico City-Mexico. E-MAIL: 5553972854.hl@gmail.com

LUZ MARÍA GARCÍA CRUZ

AdDress: Autonomous Metropolitan University, Dept. of Basic Sciences, 0200, Mexico City-Mexico. E-MAIL: lmgc@correo.azc.uam.mx

ANA ElizABETH GARCíA

AdDress: Autonomous Metropolitan University, Dept. of Basic Sciences, 0200, Mexico City-Mexico. E-MAIL: aelizagh@yahoo.com.mx 